\title{
APLICAÇÃO DO METODO DE AVALIAÇÃO DE CAPACIDADES TECNOLÓGICAS E PROCESSOS DE APRENDIZAGEM COMO FERRAMENTA GERENCIAL PARA ACUMULAÇÃO DE COMPETENCIAS E MELHORIA DE PERFORMANCE*
}

Eduardo Côrtes de Castro ${ }^{1}$

\begin{abstract}
Resumo
O presente trabalho aplica o modelo de análise de capacitação tecnológica desenvolvido por Figueiredo (2001) para definir um método de aumento de competências e melhoria de performance numa unidade de prestação de serviços da Companhia Siderúrgica Nacional, a gerência de oficina de cilindros de laminação. A utilização deste método permitiu o aumento da capacitação técnica da unidade, através dos processos de aprendizagem subjacentes, e a melhoria de performance, medida por indicadores chave de desempenho relevantes para o resultado da empresa (qualidade, custo, atendimento e produtividade da mão de obra). São apresentados, além dos resultados, a estrutura analítica do modelo proposto e as evidências associadas às capacidades tecnológicas acumuladas e aos processos de aprendizagem.
\end{abstract}

Palavras-chave: Capacidades tecnológicas; Processos de aprendizagem; Inovação tecnológica; Oficina de cilindros.

\section{APPLICATION OF THE METHOD OF EVALUATION OF TECHNOLOGICAL CAPABILITIES AND LEARNING PROCESSES AS A MANAGEMENT TOOL FOR ACCUMULATION OF COMPETENCES AND IMPROVEMENT OF PERFORMANCE}

\begin{abstract}
The present work applies the technological capabilities analysis model developed by Figueiredo (2001) to define a method of increasing competencies and improving performance in a service unit of Companhia Siderúrgica Nacional, the roll shop management. The use of this method allowed the unit's technical capacity to be increased through the underlying learning processes and performance improvement as measured by key performance indicators relevant to the company's performance (quality, cost, service and labor productivity). In addition to the results, the analytical structure of the proposed model and the evidences associated with accumulated technological capacities and learning processes are presented.

Keywords: Technological capabilities; Learning processes; Technological innovation; Roll shop.
\end{abstract}

Mestre em Administração de Empresas pela FGV-RJ, mestre em Engenharia Metalúrgica pela PUC-RJ, graduado em Engenharia Metalúrgica pela UFF, Gerente da Oficina de Cilindros da CSN, Volta Redonda, Rio de Janeiro, Brasil. 


\section{INTRODUÇÃO}

Um dos maiores desafios das empresas é criar conhecimento e inovar, aumentando sua competitividade. Embora este desafio seja maior para as empresas de economias emergentes, que precisam superar obstáculos visando a mudar seu nível de capacitação tecnológica (muitas vezes em estágio básico de desenvolvimento), empresas de economias avançadas também enfrentam esta realidade. Afinal, a sobrevivência num mercado global depende diretamente da eficiência em suas operações (menor custo, maior produtividade, maior agilidade e menor tempo de resposta às demandas externas) e do seu grau de inovação (seja em seus produtos, serviços ou gestão). Um fator chave neste contexto é a geração de conhecimento, capaz de mudar o status da empresa e torná-la mais competitiva.

Visando a explicar a trajetória de desenvolvimento das empresas, Figueiredo (2001) propôs um modelo analítico (fig. 1) que associa os processos de acumulação de capacidades tecnológicas aos de aprendizagem e, também, à melhoria de performance. Este modelo tem sido usado por autores para explicar e oferecer evidências de desenvolvimento tecnológico de setores da economia brasileira (Figueiredo, 2015).

Embora este modelo seja eficiente para descrição das estratégias adotadas pelas empresas, ele não constitui um método para que os executivos direcionem suas ações. O presente trabalho pretende ocupar esta lacuna, propondo a aplicação deste modelo como ferramenta gerencial, visando a definir estratégia corporativa, baseado na acumulação de capacidades tecnológicas e nos processos de aprendizagem.

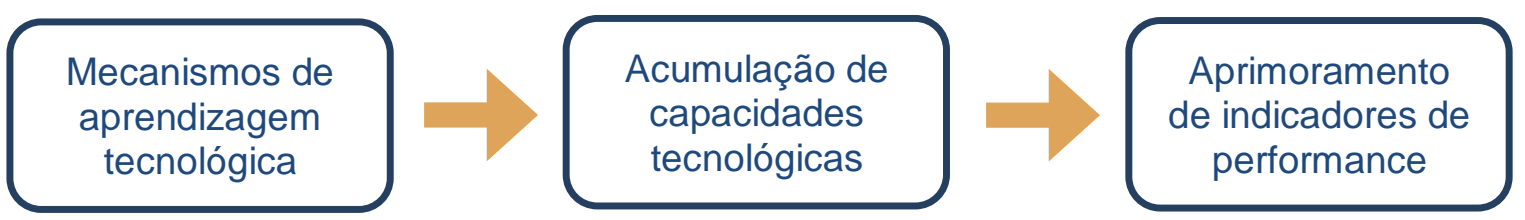

Figura 1 - Estrutura analítica do modelo proposto por Figueiredo (2001) para explicar o desenvolvimento de empresas em economias emergentes.

\section{CONTEXTO EMPÍRICO}

Este artigo baseia-se em estudo de caso individual para examinar as implicações dos processos de aprendizagem para acumulação de capacidades tecnológicas e aprimoramento de performance. Esta relação é examinada na unidade de cilindros de laminação da CSN, localizada em Volta Redonda - RJ (2012 a 2016). A CSN, criada em 1946 dentro do processo de industrialização no país, é a maior usuária nacional de cilindros de laminação em uma única planta. Sua variedade de processos e de produtos, que atendem a diversos fins (construção civil, indústria automobilística, embalagens, etc.), exige $\mathrm{o}$ uso de cilindros com diferentes características e especificações. A CSN produz especificações de aço de elevado valor agregado, como produtos laminados a quente e a frio, galvanizados e folhas de flandres.

\section{MOdELOS PARA AVALIAÇÃo DE CAPACIDADES TECNOLÓGICAS E PROCESSOS DE APRENDIZAGEM}

A trajetória de acumulação de capacidades tecnológicas na unidade de cilindros da CSN é avaliada à luz da estrutura proposta por Figueiredo (2001). Esta estrutura foi 
adaptada para unidade gerencial estudada e é apresentada na Tabela 1, onde observa-se que a acumulação de capacidades pode variar de níveis básicos (atividades de rotina) a níveis de maior grau de complexidade (atividades inovadoras). Na Tabela 1, a primeira coluna mostra os níveis de capacidade; a segunda, a função tecnológica processo de preparação de cilindros. Nas linhas estão dispostos os graus de dificuldade de cada nível de capacidade, mostrando a respectiva descrição para cada função.

Tabela 1. Capacidades tecnológicas: Unidade de Cilindros de Laminação da CSN

\begin{tabular}{|c|c|}
\hline \multirow{2}{*}{$\begin{array}{l}\text { Nível de capacidades } \\
\text { tecnológicas }\end{array}$} & PROCESSO \\
\hline & Atividades de rotina \\
\hline $\begin{array}{l}\text { Nível } 1 \\
\text { BÁSICO }\end{array}$ & $\begin{array}{l}\text { Fabricação através de processos elementares (retificação, jateamento) com } \\
\text { controle manual de equipamentos e de parâmetros produção. Registros de } \\
\text { produção feitos manualmente. }\end{array}$ \\
\hline \multirow[t]{2}{*}{$\begin{array}{l}\text { Nível } 2 \\
\text { RENOVADO }\end{array}$} & $\begin{array}{l}\text { Fabricação através de processos sofisticados (EDT), sistemas automáticos } \\
\text { para controle de equipamentos (CNC) e de parâmetros produção. } \\
\text { Certificação ISO9001 e QS9000. }\end{array}$ \\
\hline & Atividades inovadoras \\
\hline $\begin{array}{l}\text { Nível } 3 \\
\text { EXTRA BÁSICO }\end{array}$ & $\begin{array}{l}\text { Pequenas adaptações em processos, eliminação de gargalos de produção e } \\
\text { aumento da capacidade produtiva. Desenvolvimento esporádico de sistemas } \\
\text { próprios de supervisão e controle do processo de produção. }\end{array}$ \\
\hline $\begin{array}{l}\text { Nível } 4 \\
\text { PRÉ-INTERMEDIÁRIO }\end{array}$ & $\begin{array}{l}\text { Adaptações frequentes em processos e aumento sistemático da capacidade } \\
\text { produtiva. Frequentes desenvolvimentos de sistemas de supervisão e } \\
\text { controle do processo de produção. Introdução de técnicas gerenciais para } \\
\text { controle de processo (TQC, 5S, CEP, CCQ, Kaizen, Poka Yoke, MRP, ERP). }\end{array}$ \\
\hline $\begin{array}{l}\text { Nível } 5 \\
\text { INTERMEDIÁRIO }\end{array}$ & $\begin{array}{l}\text { Refino dos sistemas gerenciais de produção (TQC, 5S, CEP, CCQ, Kaizen, } \\
\text { Poka Yoke, MRP, ERP). Aprimoramento contínuo dos processos produtivos. }\end{array}$ \\
\hline $\begin{array}{l}\text { Nível } 6 \\
\text { INTERMEDIÁRIO } \\
\text { SUPERIOR }\end{array}$ & $\begin{array}{l}\text { Desenvolvimento de atividades de P\&D e Engenharia na empresa para } \\
\text { aprimoramento de processos. Associação com centros P\&D voltados para } \\
\text { atividades de inovação tecnológica em processos. Integração entre as } \\
\text { diferentes bases cognitivas organizacionais para construção de novos } \\
\text { sistemas gerenciais }\end{array}$ \\
\hline $\begin{array}{l}\text { Nível } 7 \\
\text { AVANÇADO }\end{array}$ & $\begin{array}{l}\text { Geração de técnicas organizacionais inovadoras para a indústria, baseados } \\
\text { em atividades de P\&D avançadas. Comprometimento organizacional com a } \\
\text { determinação de novos processos. Redefinição dos paradigmas tecnológicos } \\
\text { da indústria. }\end{array}$ \\
\hline
\end{tabular}

Fonte: adaptada de Figueiredo (2001), Bell e Pavitt (1995) e Lall (1992).

Empresas situadas em países em industrialização operam, sob o ponto de vista tecnológico, em condição pouco competitiva no mercado mundial. A questão básica é desenvolver capacidade tecnológica para tornar-se competitiva (Bell et al., 1984). A construção de capacidades tecnológicas e a subsequente melhoria de performance são influenciadas pelos processos de aprendizagem (Figueiredo, 2001). Este estudo propõe a adaptação da estrutura analítica desenvolvida por Figueiredo (2001) para definir a estratégia de acumulação de capacidade tecnológica por meio dos processos de aprendizagem (Tabela 2) para melhoria de performance. Na Tabela 2, estão dispostos os processos de aprendizagem, divididos em aquisição (interna ou externa) e conversão de conhecimento pela codificação ou 
socialização. As demais colunas são compostas das características-chaves dos processos de aprendizagem: variedade, intensidade, funcionamento e interação.

Tabela 2 - Processos de Aprendizagem em Empresas em Industrialização: Unidade de Cilindros de laminação da CSN

\begin{tabular}{|c|c|c|c|c|}
\hline \multirow{3}{*}{$\begin{array}{l}\text { Processos de } \\
\text { aprendizagem }\end{array}$} & \multicolumn{4}{|c|}{ Características-chave dos processos de aprendizagem } \\
\hline & Variedade & Intensidade & Funcionamento & Interação \\
\hline & Ausente-presente & $\begin{array}{l}\text { Uma vez- } \\
\text { intermitente- } \\
\text { contínuo }\end{array}$ & $\begin{array}{l}\text { Ruim-moderado- } \\
\text { bom-excelente }\end{array}$ & $\begin{array}{l}\text { Fraca-moderada- } \\
\text { forte }\end{array}$ \\
\hline \multicolumn{5}{|c|}{ Processos e mecanismos de aquisição de conhecimento } \\
\hline $\begin{array}{c}\text { Aquisição } \\
\text { externa de } \\
\text { conhecimento }\end{array}$ & $\begin{array}{l}\text { Presença/ausência de } \\
\text { processos para } \\
\text { adquirir conhecimento } \\
\text { localmente e/ou no } \\
\text { exterior. }\end{array}$ & $\begin{array}{c}\text { O modo como a } \\
\text { empresa usa este } \\
\text { processo ao longo do } \\
\text { tempo pode ser } \\
\text { contínuo, intermitente, } \\
\text { ocorrer uma vez. }\end{array}$ & $\begin{array}{c}\text { O modo como o } \\
\text { processo é criado e o } \\
\text { modo como ele opera } \\
\text { ao longo do tempo. }\end{array}$ & $\begin{array}{c}\text { Modo como um } \\
\text { processo influencia } \\
\text { outro processo de } \\
\text { aquisição externa ou } \\
\text { interna e/ou processo } \\
\text { de conversão. }\end{array}$ \\
\hline $\begin{array}{c}\text { Aquisição } \\
\text { interna de } \\
\text { conhecimento }\end{array}$ & $\begin{array}{c}\text { Presença ou ausência } \\
\text { de processos para } \\
\text { adquirir conhecimento, } \\
\text { fazendo atividades (de } \\
\text { rotina ou inovadoras) } \\
\text { internas. }\end{array}$ & $\begin{array}{c}\text { O modo como a } \\
\text { empresa usa } \\
\text { diferentes processos } \\
\text { para aquisição interna } \\
\text { de conhecimento. }\end{array}$ & $\begin{array}{l}\text { O modo como o } \\
\text { processo é criado e } \\
\text { como ele opera ao } \\
\text { longo do tempo tem } \\
\text { implicações para } \\
\text { variedade e } \\
\text { intensidade. }\end{array}$ & $\begin{array}{c}\text { Processo de } \\
\text { conhecimento interno } \\
\text { pode ser influenciado } \\
\text { por processo de } \\
\text { aquisição externa. }\end{array}$ \\
\hline \multicolumn{5}{|c|}{ Processos e mecanismos de conversão de conhecimento } \\
\hline $\begin{array}{c}\text { Socialização } \\
\text { de } \\
\text { conhecimento }\end{array}$ & $\begin{array}{l}\text { Presença/ausência de } \\
\text { diferentes processos } \\
\text { através dos quais } \\
\text { indivíduos } \\
\text { compartilham seu } \\
\text { conhecimento tácito. }\end{array}$ & $\begin{array}{c}\text { Modo como processos } \\
\text { prosseguem ao longo } \\
\text { dos anos. Intensidade } \\
\text { contínua da } \\
\text { socialização do } \\
\text { conhecimento pode } \\
\text { influenciar codificação. }\end{array}$ & \begin{tabular}{|c|} 
Modo como \\
mecanismos de \\
socialização são \\
criados/operam ao \\
longo do tempo tem \\
implicações para \\
variedade/intensidade \\
da conversão.
\end{tabular} & $\begin{array}{c}\text { Condução de } \\
\text { conhecimentos tácitos } \\
\text { para um sistema } \\
\text { efetivo. Socialização } \\
\text { pode ser influenciada } \\
\text { por processos de } \\
\text { aquisição } \\
\text { externa/interna. }\end{array}$ \\
\hline $\begin{array}{l}\text { Codificação de } \\
\text { conhecimento }\end{array}$ & $\begin{array}{l}\text { Presença/ausência de } \\
\text { diferentes processos e } \\
\text { mecanismos para } \\
\text { codificar o } \\
\text { conhecimento tácito. }\end{array}$ & $\begin{array}{l}\text { Modo como processos } \\
\text { como padronização de } \\
\text { operações são feitos. } \\
\text { Codificação ausente } \\
\text { ou intermitente limita } \\
\text { aprendizagem. }\end{array}$ & $\begin{array}{c}\text { Modo como a } \\
\text { codificação é criada e } \\
\text { opera ao longo do } \\
\text { tempo tem implicações } \\
\text { para o funcionamento } \\
\text { de todo o processo de } \\
\text { conversão. }\end{array}$ & $\begin{array}{c}\text { Modo como } \\
\text { codificação é } \\
\text { influenciada por } \\
\text { processos de } \\
\text { aquisição de } \\
\text { conhecimento ou por } \\
\text { processos de } \\
\text { socialização. }\end{array}$ \\
\hline
\end{tabular}

Fonte: Figueiredo (2001).

\section{DESENHO E ESTRATÉGIAS DO ESTUDO}

Este estudo foi estruturado para avaliar como o modelo desenvolvido por Figueiredo (2001) pode ser aplicado visando a definir a estratégia no sentido de acumular capacidades tecnológicas na unidade de cilindros da CSN relativas à função processo no período compreendido entre 2012 e 2016. Será apresentado o papel dos processos de aprendizagem no modo e velocidade de acumulação de capacidades neste período e, também, quais foram as implicações da acumulação de capacidades para a performance técnica. Para examinar estas questões com adequado nível de detalhe e profundidade, foi necessária a coleta de evidências, qualitativas e quantitativas, sobre as atividades tecnológicas e os diversos mecanismos de aprendizagem usados. Essas evidências foram obtidas a partir de várias fontes: observação direta com gerentes, engenheiros e técnicos, pesquisas em documentos (relatórios, padrões, dados históricos etc.) e coleta de dados. Utilizou-se o método de estudo de caso individual (Yin, 2001). Este método permite o exame de questões ainda não observadas na literatura em profundidade e detalhe. Logo, este estudo pretende contribuir para o aprofundamento do entendimento de 
como estratégias corporativas podem ser conduzidas a partir do modelo de avaliação de capacidades tecnológicas e dos processos de aprendizado subjacentes, visando a melhoria de performance.

\section{ANÁLISE EMPÍRICA}

\subsection{Acumulação de Capacidades Tecnológicas}

Nesta seção é apresentada a trajetória de acumulação de capacidades tecnológicas da unidade de cilindros no período de 2012 a 2016, usando a estrutura da tabela 1.

A figura 2 mostra que a unidade de cilindros da CSN acumulou capacidades inovadoras, a partir de 2013, para a função processo. Entretanto, a velocidade de acumulação de novas capacidades de cada processo variou durante o período estudado. A função processo alcançou o nível intermediário em 2016, evoluindo de atividades de rotina para atividades inovadoras.

No presente trabalho, a avaliação inicial foi realizada em 2013, quando foi constatado o nível em que a unidade de cilindros de laminação se encontrava naquele momento. À partir desta avaliação, foram identificadas as necessidades para formação de uma base cognitiva que permitisse a acumulação de novas capacidades tecnológicas. Então foram estabelecidos esforços deliberados no sentido de se desenvolver nossos conhecimentos através dos processos de aprendizagem (aquisição e conversão de conhecimento), levando-se em consideração suas características-chave (variedade, intensidade, funcionamento e interação).

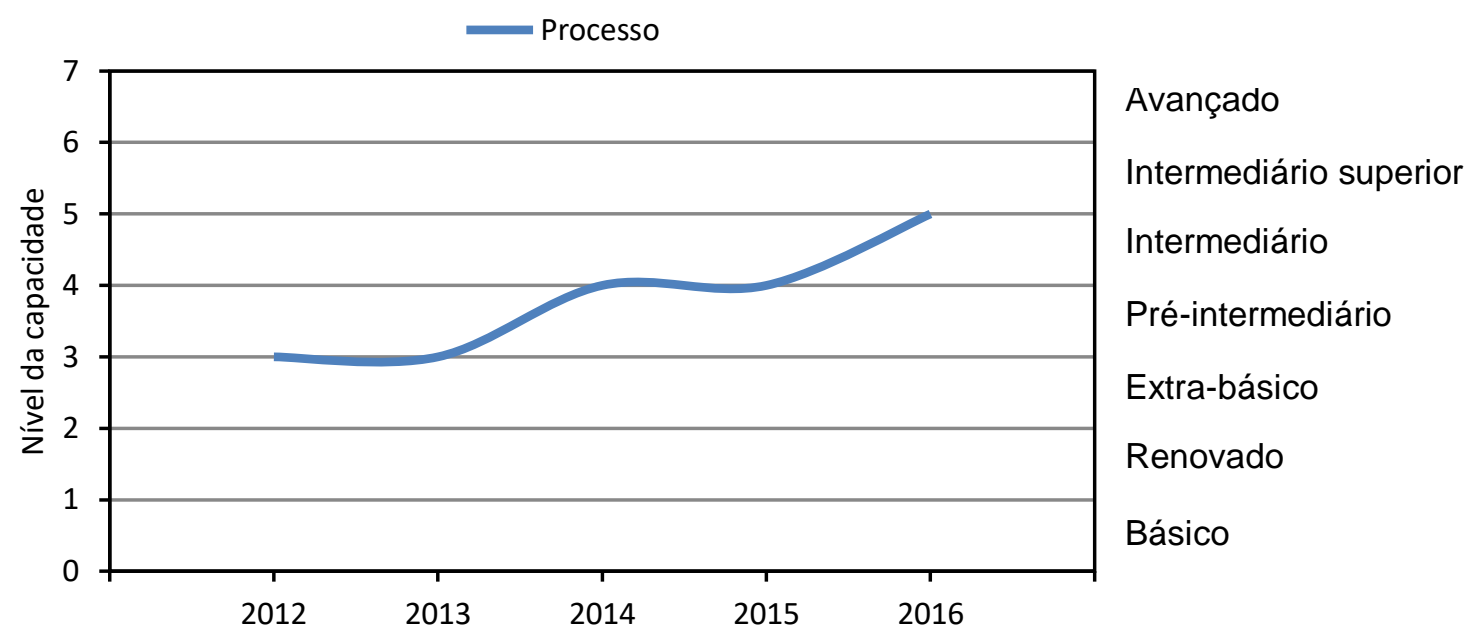

Figura 2. Trajetória de Acumulação de Capacidades Tecnológicas na Unidade de Cilindros da CSN para a Função Processo (2012 a 2016).

\subsection{Processos de Aprendizagem}

\subsubsection{Variedade dos Processos de Aprendizagem}

A Tabela 3 mostra a variedade de mecanismos empregados pela unidade de cilindros da CSN em cada processo de aprendizagem. As evidências apontam que a acumulação de capacidades tecnológicas na unidade foi acompanhada pelo aumento progressivo na variedade de mecanismos de aquisição e conversão do conhecimento.

Em 2012, havia 30 mecanismos de aquisição e conversão. Nesse ano, a unidade encontrava-se limitada em relação ao uso eficiente dos processos de aprendizagem. Ou seja, não havia uma boa sintonia entre as características chave. 
Tabela 3: Variedade dos Processos de Aprendizagem Usados pela Unidade de cilindros da CSN

\begin{tabular}{|c|c|c|c|c|c|c|}
\hline & Processo de aprendizagem & 2012 & 2013 & 2014 & 2015 & 2016 \\
\hline \multirow{18}{*}{ 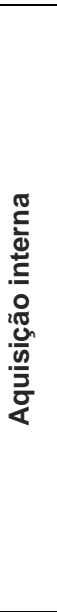 } & Usina do Conhecimento & Presente & Presente & Presente & Presente & Presente \\
\hline & Comitê de análise de falha & Ausente & Ausente & Presente & Presente & Presente \\
\hline & Análise semanal & Ausente & Presente & Presente & Presente & Presente \\
\hline & Reuniões de projetos & Ausente & Ausente & Presente & Presente & Presente \\
\hline & Treinamento Laser Tracker & Ausente & Ausente & Presente & Presente & Presente \\
\hline & Processo de seleção & Ausente & Ausente & Presente & Presente & Presente \\
\hline & Rotação posto de trabalho & Ausente & Ausente & Presente & Presente & Presente \\
\hline & Utilização Laser Tracker & Ausente & Ausente & Ausente & Presente & Presente \\
\hline & Seminário interno & Ausente & Ausente & Ausente & Presente & Presente \\
\hline & Comitê gestão de custo & Ausente & Ausente & Ausente & Ausente & Presente \\
\hline & Auditorias de processo & Ausente & Ausente & Ausente & Presente & Presente \\
\hline & Ativo 10 & Presente & Presente & Presente & Presente & Presente \\
\hline & Mapeamento de processo & Ausente & Ausente & Presente & Presente & Presente \\
\hline & Gestão ISO/TS & Ausente & Presente & Presente & Presente & Presente \\
\hline & Estágio interno & Presente & Presente & Presente & Presente & Presente \\
\hline & Cursos internos & Presente & Presente & Presente & Presente & Presente \\
\hline & Rotinas de produção & Presente & Presente & Presente & Presente & Presente \\
\hline & Multifuncionalidade & Presente & Presente & Presente & Presente & Presente \\
\hline \multirow{15}{*}{ 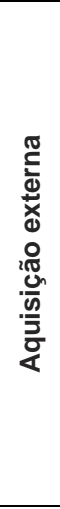 } & Seminários, congressos, etc. & Ausente & Presente & Presente & Presente & Presente \\
\hline & Interação fornecedores & Presente & Presente & Presente & Presente & Presente \\
\hline & Cursos com fornecedores & Presente & Presente & Presente & Presente & Presente \\
\hline & Treinamento Laser Tracker & Ausente & Ausente & Presente & Presente & Ausente \\
\hline & Interação com clientes & Presente & Presente & Presente & Presente & Presente \\
\hline & Projetos com clientes & Presente & Presente & Presente & Presente & Presente \\
\hline & Projetos com fornecedores & Presente & Presente & Presente & Presente & Presente \\
\hline & Cursos (mestrado, MBA, etc) & Ausente & Ausente & Ausente & Presente & Presente \\
\hline & Curso superior & Presente & Presente & Presente & Presente & Presente \\
\hline & Curso técnico & Presente & Presente & Presente & Presente & Presente \\
\hline & Estágio externo & Presente & Presente & Presente & Presente & Presente \\
\hline & Programa Capacitar & Presente & Presente & Presente & Presente & Presente \\
\hline & Programa Jovem Aprendiz & Presente & Presente & Presente & Presente & Presente \\
\hline & Consulta à literatura & Presente & Presente & Presente & Presente & Presente \\
\hline & Programa Escola de Líderes & Presente & Presente & Presente & Presente & Presente \\
\hline \multirow{13}{*}{ 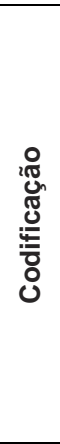 } & Padronização & Presente & Presente & Presente & Presente & Presente \\
\hline & Relatório análise de falha & Ausente & Ausente & Presente & Presente & Presente \\
\hline & Relatórios técnicos & Presente & Presente & Presente & Presente & Presente \\
\hline & Trabalhos seminário interno & Ausente & Ausente & Ausente & Presente & Presente \\
\hline & Relatórios de turno & Ausente & Ausente & Presente & Presente & Presente \\
\hline & Rondas processo & Ausente & Ausente & Presente & Presente & Presente \\
\hline & Check lists de processo & Ausente & Ausente & Presente & Presente & Presente \\
\hline & Ordens de serviço & Ausente & Ausente & Presente & Presente & Presente \\
\hline & Requisição serviço manutenção & Presente & Presente & Presente & Presente & Presente \\
\hline & Análise semanal & Ausente & Presente & Presente & Presente & Presente \\
\hline & Relatório FMEA & Ausente & Ausente & Ausente & Ausente & Presente \\
\hline & Programa 5 "S" & Presente & Presente & Presente & Presente & Presente \\
\hline & Mapeamento de processo & Ausente & Ausente & Presente & Presente & Presente \\
\hline \multirow{15}{*}{ 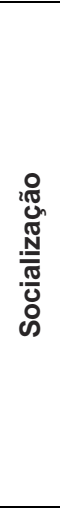 } & Reunião de análise de falha & Ausente & Ausente & Presente & Presente & Presente \\
\hline & Interação com fornecedores & Presente & Presente & Presente & Presente & Presente \\
\hline & Reunião de análise semanal & Ausente & Presente & Presente & Presente & Presente \\
\hline & Reuniões de projetos & Ausente & Ausente & Presente & Presente & Presente \\
\hline & Interação com clientes & Presente & Presente & Presente & Presente & Presente \\
\hline & Utilização Laser Tracker & Ausente & Ausente & Ausente & Presente & Presente \\
\hline & Treinamento posto de trabalho & Presente & Presente & Presente & Presente & Presente \\
\hline & Diagnóstico trabalho operacional & Presente & Presente & Presente & Presente & Presente \\
\hline & Participação seminário interno & Ausente & Ausente & Ausente & Presente & Presente \\
\hline & Comitê de gestão de custo & Ausente & Ausente & Ausente & Ausente & Presente \\
\hline & Grupos de trabalho & Presente & Presente & Presente & Presente & Presente \\
\hline & Auditorias internas de qualidade & Presente & Presente & Presente & Presente & Presente \\
\hline & Interação staff e operador & Presente & Presente & Presente & Presente & Presente \\
\hline & Capacitar novos colaboradores & Presente & Presente & Presente & Presente & Presente \\
\hline & Capacitar estagiário/aprendiz & Presente & Presente & Presente & Presente & Presente \\
\hline
\end{tabular}


Fonte: elaboração própria do autor

Tabela 3: Variedade dos Processos de Aprendizagem Usados pela Unidade de cilindros da CSN

\begin{tabular}{|l|c|c|c|c|c|}
\hline Processo de aprendizagem & $\mathbf{2 0 1 2}$ & $\mathbf{2 0 1 3}$ & $\mathbf{2 0 1 4}$ & $\mathbf{2 0 1 5}$ & $\mathbf{2 0 1 6}$ \\
\hline Subtotal aquisição interna & Limitada & Moderada & Diversa & Diversa & Diversa \\
\hline Subtotal aquisição externa & Diversa & Diversa & Diversa & Diversa & Diversa \\
\hline Subtotal padronização & Limitada & Limitada & Diversa & Diversa & Diversa \\
\hline Subtotal socialização & Moderada & Diversa & Diversa & Diversa & Diversa \\
\hline Total de mecanismos & 31 & 36 & 51 & 58 & 60 \\
\hline
\end{tabular}

Fonte: elaboração própria do autor

Em 2013, houve o acréscimo de 5 mecanismos. Os novos mecanismos permitiram acumular novas capacidades, especialmente na adaptação de alguns sobressalentes, descontinuados no mercado, necessários para o bom funcionamento de algumas retificadoras de cilindros. Um aspecto que requereu uma atenção especial neste período (e que se tornaria mais crítico) foi o turn over, que representou uma renovação na mão de obra (substituição de colaboradores aposentados, com maior experiência). A principal forma de conversão de conhecimento foi a organização da gestão dos resultados, o que foi feito através da definição de indicadores chave, de metas e de reuniões sistemáticas com os supervisores e o staff. Este mecanismo permitiu ao time maior coesão em torno de um objetivo comum.

Em 2014, houve um salto significativo no número de mecanismos usados, passando de 35 para 50. O acréscimo ocorreu em todos os mecanismos. Neste ano, foi estruturado um comitê para registro e análise de falhas (conhecido internamente como FRAF). Todos os técnicos, engenheiros e supervisores fizeram curso para conhecer métodos de trabalho para identificação de problemas, suas causas e ferramentas voltadas para neutralização através de ações corretivas eficazes. Esta ferramenta permitiu solucionar problemas pontuais. Os problemas crônicos foram tratados através de método para análise e solução de problemas (MASP). Ou seja, projetos de médio ou longo prazos. Estes mecanismos (FRAF e MASP) desencadearam outros com efeito capilar até os níveis operacionais, permitindo, além da consolidação das ações corretivas, a aplicação de ações preventivas. A maior capacitação da equipe permitiu a melhora na qualidade dos cilindros produzidos, reduzindo as falhas e os defeitos e melhorando o desempenho dos mesmos nos laminadores.

Em 2015, havia 57 mecanismos. Os novos mecanismos se agregaram àqueles criados em 2014 e ajudaram a consolidar os conhecimentos adquiridos e compartilhados. Foram criadas auditorias internas de processo, que visavam a identificar potenciais falhas, tomando ação antes que um problema ocorresse. Outro mecanismo muito importante foi a criação de um seminário interno de projetos. Neste seminário, os principais trabalhos da unidade são compartilhados por toda a equipe. Um dos frutos destes trabalhos foi a capacitação da equipe no uso do equipamento conhecido como laser tracker, um dispositivo eletrônico que faz medidas dimensionais em peças e equipamentos mecânicos. O domínio desta técnica permitiu a análise com elevada precisão (e produtividade) das condições dos laminadores, auxiliando as equipes de manutenção nas decisões sobre intervenção nos equipamentos, antes da ocorrência de falhas que comprometessem os resultados de seus processos.

Em 2016, havia 59 mecanismos. Neste ano, o principal mecanismo de aquisição de conhecimento criado foi a aplicação do método de prevenção de falhas (FMEA - 
Failure Mode and Effect Analysis). Método implantado com o objetivo de ampliar o uso de ferramentas preventivas. Ou seja, trabalhar com mais foco em melhoria de performance. As equipes aumentaram a confiabilidade de suas atividades. A aplicação desse método, associado aos mecanismos acumulados até então (principalmente a interação com clientes e fornecedores), permitiu que fossem adquiridas capacidades tecnológicas em processo, contribuindo para a redução do consumo de cilindros.

\subsubsection{Intensidade dos Processos de Aprendizagem}

A Tabela 4 mostra a intensidade dos processos de aprendizagem entre 2012 e 2016. Em 2012, foram adotados alguns mecanismos isolados para aquisição interna, codificação e socialização. Para a aquisição de conhecimento externo, que teve maior intensidade neste período, usou-se mecanismos associados aos programas corporativos de capacitação da CSN (Capacitar, Jovem Aprendiz, Escola de Líderes e estágios); e a interação com fornecedores e com clientes.

Em 2013, houve pouca alteração quando comparado com 2012. A intensidade aumentou apenas devido ao aumento no número de mecanismos. Mas a partir de 2014, a intensidade aumentou de forma sensível. A reorganização realizada no sistema de gestão da unidade, baseada na definição de indicadores chave, estabelecimento de metas, estruturação e capacitação de equipes multidisciplinares voltadas para melhoria dos processos (comitê de tratamento de anomalias e projetos de melhoria), além da aplicação dos mecanismos de conversão do conhecimento desde o nível do staff até o nível operacional, permitiram o aporte de novos conhecimentos. Um fator importante neste período foi a mudança ocorrida no processo de seleção para mudança de cargos. As promoções passaram a ser feitas com base em avaliações teóricas e práticas para todos os postos da unidade. Com isso, os colaboradores se motivaram a estudar e a ampliar seus conhecimentos.

Tabela 4: Intensidade dos Processos de Aprendizagem Usados pela Unidade de cilindros da CSN

\begin{tabular}{|c|c|c|c|c|c|c|}
\hline & Processo de aprendizagem & 2012 & 2013 & 2014 & 2015 & 2016 \\
\hline \multirow{18}{*}{ 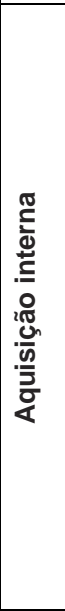 } & Usina do Conhecimento & Baixa & Baixa & Intemit. & Intemit. & Intemit. \\
\hline & Comitê de análise de falha & - & - & Contínua & Contínua & Contínua \\
\hline & Análise semanal & - & Contínua & Contínua & Contínua & Contínua \\
\hline & Reuniões de projetos & - & - & Contínua & Contínua & Contínua \\
\hline & Treinamento Laser Tracker & - & - & Intemit. & Intemit. & Intemit. \\
\hline & Processo de seleção & - & - & Contínua & Contínua & Contínua \\
\hline & Rotação posto de trabalho & - & - & Baixa & Intemit. & Contínua \\
\hline & Utilização Laser Tracker & - & - & - & Intemit. & Contínua \\
\hline & Seminário interno & - & - & - & Contínua & Contínua \\
\hline & Comitê gestão de custo & - & - & - & - & Contínua \\
\hline & Auditorias de processo & - & - & - & Contínua & Contínua \\
\hline & Ativo 10 & Baixa & Baixa & Baixa & Baixa & Baixa \\
\hline & Mapeamento de processo & - & - & Intemit. & Contínua & Contínua \\
\hline & Gestão ISO/TS & - & Baixa & Intemit. & Contínua & Contínua \\
\hline & Estágio interno & Baixa & Baixa & Intemit. & Contínua & Contínua \\
\hline & Cursos internos & Baixa & Baixa & Baixa & Baixa & Baixa \\
\hline & Rotinas de produção & Contínua & Contínua & Contínua & Contínua & Contínua \\
\hline & Multifuncionalidade & Baixa & Intemit. & Contínua & Contínua & Contínua \\
\hline \multirow{9}{*}{ 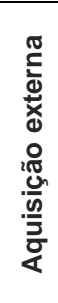 } & Seminários, congressos, etc. & - & Baixa & Baixa & Baixa & Baixa \\
\hline & Interação fornecedores & Contínua & Contínua & Contínua & Contínua & Contínua \\
\hline & Cursos com fornecedores & Contínua & Contínua & Contínua & Contínua & Contínua \\
\hline & Treinamento Laser Tracker & - & - & Contínua & Contínua & - \\
\hline & Interação com clientes & Contínua & Contínua & Contínua & Contínua & Contínua \\
\hline & Projetos com clientes & Baixa & Baixa & Intemit. & Intemit. & Contínua \\
\hline & Projetos com fornecedores & Contínua & Contínua & Contínua & Contínua & Contínua \\
\hline & Cursos (mestrado, MBA, etc) & - & - & - & Baixa & Baixa \\
\hline & Curso superior & Intemit. & Intemit. & Intemit. & Intemit. & Intemit. \\
\hline
\end{tabular}




\begin{tabular}{|l|l|l|l|l|l|l|}
\hline & Curso técnico & Contínua & Contínua & Contínua & Contínua & Contínua \\
\cline { 2 - 6 } & Estágio externo & Contínua & Contínua & Contínua & Contínua & Contínua \\
\cline { 2 - 6 } & Programa Capacitar & Contínua & Contínua & Contínua & Contínua & Contínua \\
\cline { 2 - 6 } & Programa Jovem Aprendiz & Contínua & Contínua & Contínua & Contínua & Contínua \\
\cline { 2 - 6 } & Consulta à literatura & Contínua & Contínua & Contínua & Contínua & Contínua \\
\cline { 2 - 5 } & Programa Escola de Líderes & Contínua & Contínua & Contínua & Contínua & Contínua \\
\hline
\end{tabular}

Fonte: elaboração própria do autor

Tabela 4: Intensidade dos Processos de Aprendizagem Usados pela Unidade de cilindros da CSN

\begin{tabular}{|c|c|c|c|c|c|c|}
\hline & Processo de aprendizagem & 2012 & 2013 & 2014 & 2015 & 2016 \\
\hline \multirow{13}{*}{ 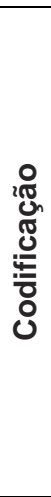 } & Padronização & Contínua & Contínua & Contínua & Contínua & Contínua \\
\hline & Relatório análise de falha & - & - & Contínua & Contínua & Contínua \\
\hline & Relatórios técnicos & Intemit. & Intemit. & Intemit. & Intemit. & Intemit. \\
\hline & Trabalhos seminário interno & - & - & - & Contínua & Contínua \\
\hline & Relatórios de turno & - & - & Contínua & Contínua & Contínua \\
\hline & Rondas processo & - & - & Intemit. & Contínua & Contínua \\
\hline & Check lists de processo & - & - & Intemit. & Contínua & Contínua \\
\hline & Ordens de serviço & - & - & Intemit. & Contínua & Contínua \\
\hline & Requisição serviço manutenção & Contínua & Contínua & Contínua & Contínua & Contínua \\
\hline & Análise semanal & - & Contínua & Contínua & Contínua & Contínua \\
\hline & Relatório FMEA & - & - & - & - & Intemit. \\
\hline & Programa 5 "S" & Intemit. & Intemit. & Intemit. & Intemit. & Intemit. \\
\hline & Mapeamento de processo & - & - & Intemit. & Contínua & Contínua \\
\hline \multirow{15}{*}{ 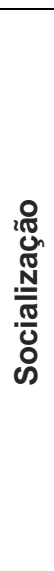 } & Reunião de análise de falha & - & - & Contínua & Contínua & Contínua \\
\hline & Interação com fornecedores & Contínua & Contínua & Contínua & Contínua & Contínua \\
\hline & Reunião de análise semanal & - & Contínua & Contínua & Contínua & Contínua \\
\hline & Reuniões de projetos & - & - & Contínua & Contínua & Contínua \\
\hline & Interação com clientes & Contínua & Contínua & Contínua & Contínua & Contínua \\
\hline & Utilização Laser Tracker & - & - & - & Contínua & Contínua \\
\hline & Treinamento posto de trabalho & Intemit. & Intemit. & Intemit. & Intemit. & Intemit. \\
\hline & Diagnóstico trabalho operacional & Contínua & Contínua & Contínua & Contínua & Contínua \\
\hline & Participação seminário interno & - & - & - & Contínua & Contínua \\
\hline & Comitê de gestão de custo & - & - & - & - & Contínua \\
\hline & Grupos de trabalho & Intemit. & Intemit. & Intemit. & Intemit. & Intemit. \\
\hline & Auditorias internas de qualidade & Intemit. & Contínua & Contínua & Contínua & Contínua \\
\hline & Interação staff e operador & Contínua & Contínua & Contínua & Contínua & Contínua \\
\hline & Capacitar novos colaboradores & Contínua & Contínua & Contínua & Contínua & Contínua \\
\hline & Capacitar estagiário/aprendiz & Contínua & Contínua & Contínua & Contínua & Contínua \\
\hline
\end{tabular}

Fonte: elaboração própria do autor

Nos anos de 2015 e de 2016, foram consolidados os mecanismos de aquisição e conversão do conhecimento, tanto pelo aumento da intensidade dos processos já existentes quanto pela introdução de novos processos, que foram usados de forma contínua na unidade de cilindros. Foram empreendidos esforços deliberados no sentido de se trabalhar com elevada intensidade nos processos de aprendizagem identificados como mais influentes na capacitação das equipes em todos os níveis. Ou seja, visando sempre a transformar o conhecimento individual em capacidade tecnológica organizacional. As implicações destes esforços sobre os resultados foram diretas, melhorando ainda mais a qualidade dos cilindros, o índice de paradas dos laminadores, o consumo de cilindros e a produtividade da mão de obra.

\subsubsection{Funcionamento dos Processos de Aprendizagem}

O funcionamento, apresentado na Tabela 5, foi avaliado conforme critérios propostos por Figueiredo (2001). O funcionamento de cada mecanismo mostra a efetividade com que cada um influenciou a acumulação de capacidade tecnológica. Observa-se pelos resultados alcançados que houve melhora no funcionamento dos mecanismos empregados de forma contínua, sugerindo que estes mecanismos se tornaram mais eficazes para acumulação de novas capacidades e melhoria contínua de desempenho. Os mecanismos usados tiveram melhora contínua em seus 
funcionamentos e visavam a concentrar esforços para operacionalizar mudanças técnicas com o aperfeiçoamento dos processos ou equipamentos.

Tabela 5: Funcionamento dos Processos de Aprendizagem Usados pela Unidade de Cilindros

\begin{tabular}{|l|c|c|c|c|c|}
\hline Processo de aprendizagem & $\mathbf{2 0 1 2}$ & $\mathbf{2 0 1 3}$ & $\mathbf{2 0 1 4}$ & $\mathbf{2 0 1 5}$ & $\mathbf{2 0 1 6}$ \\
\hline Aquisição interna & Ruim & Ruim/mod. & Moderado & Mod./bom & Bom \\
\hline Aquisição externa & Moderado & Moderado & Bom & Bom & Bom \\
\hline Codificação & Ruim & Ruim/mod. & Moderado & Bom & Bom \\
\hline Socialização & Moderado & Mod./bom & Mod./bom & Bom & Bom \\
\hline
\end{tabular}

Fonte: elaboração própria do autor

O bom funcionamento das ações de capacitação dos colaboradores, como por exemplo as ferramentas de gestão dos resultados (estruturação de indicadores chave, auditorias internas, projetos de melhoria, análise de falhas, relatórios de turno), implantadas a partir de 2014, foram fundamentais para a difusão do conhecimento em todos os níveis e o direcionamento dos esforços para a melhoria dos resultados. A interação com fornecedores e clientes, presente durante todo 0 período avaliado e que resultavam em projetos conjuntos, permitiram que novos conhecimentos fossem assimilados pelos indivíduos e convertidos para a organização. Como resultado, os processos foram aprimorados, implicando na melhoria de todos os indicadores.

\subsubsection{Interação dos Processos de Aprendizagem}

A avaliação da interação entre e dentro dos processos de aprendizagem (Tabela 6) seguiu os critérios apresentados na seção 3 .

O presente trabalho procurou dar um foco especial aos processos de aprendizagem para o aumento do nível de capacidades tecnológicas da unidade. A gestão dos conhecimentos foi direcionada de forma que houvesse o máximo de interação entre os processos de aprendizagem. $O$ aumento contínuo da integração dos processos de aprendizagem, obtido pelo sistema de gerenciamento das rotinas e das melhorias, permitiu que a capacidade técnica fosse aprimorada ao longo do tempo. Um fator que contribuiu para isso foi o efeito multiplicador dos processos usados. A implantação de indicadores chave e suas metas (aquisição interna); as reuniões da análise de resultados, de análise de falhas e de projetos (codificação e socialização); os relatórios de desempenho, de análise de falha, de projeto, de turno e a padronização (codificação); associados à interação com fornecedores e clientes (aquisição externa); desencadeou outras iniciativas, como cursos internos e externos, desenvolvimento de novas técnicas e, principalmente, a troca de conhecimentos tácitos, que retroalimenta todo este sistema e tem sido aperfeiçoado continuamente. Um aspecto que também contribuiu para a maior interação entre os processos de aprendizagem foi o aumento da variedade e a melhora contínua do funcionamento dos mecanismos usados. No gráfico 4 visualiza-se esta transformação ao longo do tempo.

Tabela 6: Interação Entre os Processos de Aquisição e Conversão

\begin{tabular}{|l|c|c|c|c|c|c|}
\hline \multirow{2}{*}{$\begin{array}{l}\text { Processo de } \\
\text { aprendizagem }\end{array}$} & $\mathbf{2 0 1 2}$ & $\mathbf{2 0 1 3}$ & 2014 & 2015 & 2016 \\
\cline { 2 - 6 } & \multicolumn{4}{|c|}{ Aquisição de conhecimento } \\
\hline Aquisição interna & Fraca & Moderada & Moderada & Moderada/forte & Forte \\
\hline Aquisição externa & Fraca & Fraca & Moderada & Moderada & Moderada \\
\hline
\end{tabular}




\begin{tabular}{|l|l|l|l|l|l|}
\hline & \multicolumn{5}{|c|}{ Conversão de conhecimento } \\
\hline Codificação & Fraca & Moderada & Moderada & Forte & Forte \\
\hline Socialização & Fraca & Moderada & Moderada & Moderada/forte & Forte \\
\hline
\end{tabular}

Fonte: elaboração própria do autor

\section{IMPLICAÇÕES DA ACUMULAÇÃO DE CAPACIDADES TECNOLÓGICAS PARA O APRIMORAMENTO DA PERFORMANCE}

Os indicadores de performance utilizados neste trabalho são os mesmos avaliados nas reuniões de gerenciamento da unidade. Eles representam os fatores mais importantes para o sucesso no atendimento aos clientes e abrangem as seguintes dimensões da qualidade total: qualidade intrínseca, custo e entrega. Os indicadores avaliados são: qualidade - índice de desvio; custo - consumo de cilindros; entrega paradas dos clientes por responsabilidade da unidade e produtividade da mão de obra.

Os indicadores acima são apresentados sob forma de índice, cuja a base (100) é o valor de 2012 (exceto o índice de desvio, cuja base é 2013). Estes indicadores são calculados originalmente das seguintes formas: (1) índice de desvio - número de cilindros com defeito dividido pelo número total de cilindros auditados; (2) consumo de cilindros - quantidade de cilindro removido, em $\mathrm{mm}$, durante retificação nas retificadoras dividido pela produção dos laminadores; (3) parada de clientes - tempo total de parada dos clientes por responsabilidade da unidade dividido pelo tempo total programado para os laminadores produzirem; (4) produtividade da mão de obra - produção, em t, dos laminadores dividida pelo consumo de cilindros, em mm, dividida pelo número de colaboradores da unidade de cilindros.

Tabela 7: Indicadores de Performance Operacional da Unidade de Cilindros

\begin{tabular}{|c|c|c|c|c|c|c|}
\hline Indicador & Melhor & $\mathbf{2 0 1 2}$ & $\mathbf{2 0 1 3}$ & $\mathbf{2 0 1 4}$ & $\mathbf{2 0 1 5}$ & $\mathbf{2 0 1 6}$ \\
\hline $\begin{array}{c}\text { Índice de desvio de qualidade } \\
\text { (\% desvio) }\end{array}$ & $\boldsymbol{7}$ & - & 100 & 47 & 27 & 25 \\
\hline $\begin{array}{c}\text { Consumo de cilindros } \\
\text { (mm cilindro gasto/kt laminada) }\end{array}$ & $\boldsymbol{7}$ & 100 & 103 & 109 & 100 & 93 \\
\hline $\begin{array}{c}\text { Parada de clientes } \\
\text { (tempo parada/tempo disponível) }\end{array}$ & $\mathbf{7}$ & 100 & 113 & 99 & 64 & 51 \\
\hline $\begin{array}{c}\text { Produtividade mão de obra } \\
\text { (t laminada/mm cilindro)/colaborador }\end{array}$ & $\mathbf{4}$ & 100 & 103 & 105 & 109 & 105 \\
\hline
\end{tabular}

Fonte: elaboração própria do autor

A acumulação do Nível 4 de capacidades para processo permitiu novos aumentos da produtividade da mão de obra (5\% em 2014 e 9\% em 2015), melhora na qualidade dos cilindros (redução dos desvios em 53\% em 2014 e 73\% em 2015) e a redução da parada de clientes (queda de 36\% em 2015). Além da melhora na intensidade e no funcionamento da gestão dos indicadores de desempenho e na análise de falhas, o novo processo de seleção (com provas teórica e prática) propiciou o aumento da capacitação da equipe ocorrida pela promoção de colaboradores melhores qualificados e, também, o maior interesse da equipe em se desenvolver (estarem preparados) para futuras oportunidades. A codificação do conhecimento foi melhorada através da realização do mapeamento de todos os processos da unidade e, consequentemente, a revisão de procedimentos obsoletos e a criação de novos padrões operacionais. Com isso, aumentou-se a eficiência 
operacional devido ao melhor controle do processo e redução das falhas. A melhora no índice de desvio de qualidade de cilindros estava associada também ao desenvolvimento de iniciativas específicas para este fim. A identificação e solução precisa de problemas pontuais pelo uso de técnicas de análise de falhas; e de problemas crônicos pelo emprego do método de análise e solução de problemas (MASP), redundou em procedimentos operacionais mais eficazes e acompanhamentos sistemáticos (auditorias internas) por parte dos líderes de turno e dos técnicos de processo. Desta forma, qualquer desvio encontrado era solucionado rapidamente e o conhecimento gerado se consolidava de forma estruturada (padrão) e tácita (habilidades específicas). Como resultado, houve acumulação de capacitação organizacional pela aplicação de técnicas gerenciais mais eficientes (análise de falhas, auditorias de processo, rastreabilidade de dados técnicos), capacidade de identificar problemas e codificar melhores práticas.

A acumulação do Nível 5 de capacidades para processo teve implicações positivas sobre o consumo de cilindros (redução de $7 \%$ ) e a parada de clientes (queda de $49 \%$ ). A rotatividade planejada da mão de obra nos níveis técnico e de supervisão permitiu que a experiência na técnica de retífica de cilindros fosse compartilhada na oficina que ainda não tinha alcançado bons resultados e, assim, a qualidade dos cilindros produzidos neste setor melhorasse. $\mathrm{O}$ tratamento deste indicador de forma sistemática nas reuniões de análise semanal ajudou a consolidar os resultados alcançados e a aperfeiçoar as melhores práticas da unidade. Os trabalhos realizados em conjunto com os clientes e com os fornecedores de cilindros permitiram o aprimoramento do uso dos cilindros, reduzindo seu consumo. Os ajustes nas especificações dos cilindros e nos processos dos laminadores, fruto das análises de falha e dos projetos realizados com clientes e fornecedores, reduziu os acidentes e os desgastes prematuros dos cilindros. Todo o conhecimento gerado foi consolidado nos relatórios de análise de falha, nos padrões e integraram o FMEA (ferramenta preventiva de falhas). A otimização do uso dos rebolos, componente do equipamento que retifica os cilindros, obtida através de projeto específico para este fim e desenvolvido em conjunto com fornecedores deste dispositivo, também contribuiu para a redução no consumo de cilindros. A melhora na intensidade e no funcionamento dos processos de análise de falhas e dos projetos desenvolvidos com os clientes permitiu que houvesse redução na parada dos clientes por responsabilidade da unidade de cilindros. Outro aspecto importante e que merece destaque é o aumento na interação entre os processos de aprendizagem. As conexões estabelecidas entre os processos de aquisição e de conversão do conhecimento ajudaram a sedimentar a base cognitiva da unidade e a melhorar o resultado de todos os indicadores avaliados neste trabalho.

\section{CONCLUSÃO}

Este trabalho contribui para o entendimento de como o modelo desenvolvido por Figueiredo (2001) pode ser usado para estabelecimento de uma estratégia de acumulação de capacidades tecnológicas a partir dos processos de aprendizagem subjacentes. As evidências sugerem que a melhoria de performance técnica da unidade de cilindros de laminação foi influenciada pela acumulação de capacidades tecnológicas. As evidências apresentadas permitem concluir o que segue.

- A formação de uma base cognitiva permite a acumulação de capacidades tecnológicas inovadoras. A melhora nos indicadores de performance teve relação 
direta com as capacidades acumuladas, aprimoradas a partir dos processos de aprendizagem subjacentes.

- A melhora sistemática das características chave dos processos de aprendizagem (variedade, intensidade, funcionamento e interação) desempenhou um papel fundamental na acumulação de capacidades tecnológicas inovadoras e na melhora da performance da unidade de cilindros da CSN. O planejamento e o acompanhamento sistemáticos dos mecanismos de aquisição e conversão do conhecimento permitem avaliar continuamente seus resultados e a manutenção ou correção na abordagem da estratégia de desenvolvimento da capacitação técnica individual e organizacional.

- O modelo desenvolvido por Figueiredo (2001) pode ser usado como ferramenta gerencial para definição do planejamento estratégico de uma unidade produtiva, permitindo a definição de mecanismos para acumulação de capacidades tecnológicas a partir dos processos de aprendizagem.

\section{REFERÊNCIAS}

1 Figueiredo, P. N. Technological Learning and Competitive Performance. Cheltenham: Edward Elgar, 2001.

2 Figueiredo, P. N. Gestão da Inovação: Conceitos, Métricas e Experiências de Empresas no Brasil. Rio de Janeiro: LTC, 2015.

3 Bell, M., Pavitt, K. The Development of Technological Capabilities. Haque, I. U. (Ed.), Trade, Technology and International Competitiveness. Washington: The World Bank, 1995.

4 Lall, S. Technological Capabilities and Industrialization. World Development. V. 20, n. 2, p. 165-186, 1992.

5 Bell, M., Ross-Larson, B., Westphal, L. E. Assessing the Performance of the Infant Industries. Washington DC: World Bank, 1984 (World Bank Staff Working Papers, n. 666).

6 Yin, R. K. Estudo de Caso: Planejamento e Métodos. Porto Alegre: Bookman, 2001 